\title{
EARLY CLINICAL EFFECTIVENESS OF ER:YAG LASER IN ASSOCIATION WITH THE RED COMPLEX OF BACTERIA IN THE INITIAL TREATMENT OF MODERATE CHRONIC PERIODONTITIS
}

\author{
BI. Yaneva ${ }^{1}$, E. Firkova ${ }^{1}$ and E. Karaslavova ${ }^{2}$ \\ ${ }^{1}$ Department of Periodontology and Oral Diseases, Faculty of Dental Medicine, \\ Medical University - Plovdiv \\ ${ }^{2}$ Faculty of Biology, Plovdiv University "Paisii Hilendarski"
}

Summary. Chronic periodontitis is a result of polymicrobial infection and its treatment aims removal of dental calculus and biofilm from the periodontal pocket. Many hand and power-driven instruments are created for this purpose. In recent years, lasers are widely discussed tools for periodontal therapy. The aim of the present study is to evaluate the early microbiological and clinical effectiveness of the Er:YAG laser in the treatment of chronic periodontitis. 30 patients with moderate chronic periodontitis were enrolled in the study. Using a split-mouth design they were treated either with Er:YAG laser (chisel tip, $100 \mathrm{~mJ}, 15 \mathrm{~Hz}, 5-6$ water spray)-test group or with Gracey curettes- control group. Probing pocket depth (PPD), gingival recession (GR), clinical attachment level (CAL), bleeding on probing and plaque presence were evaluated at baseline and one month after the therapy. Microbiological samples were taken from the deepest four pockets of each quadrant from 20 randomly selected patients $(n=$ 80 ). They were evaluated using real time PCR for periodontal pathogens from the red complex (Porphyromonas gingivalis, Treponema denticola and Tannerella forsythia). One month after therapy in the control group PPD decreased from $4.59 \mathrm{~mm}$ to 3.36 $\mathrm{mm}$, the CAL gain was $1.09 \mathrm{~mm}$ and the reduction of bleeding and plaque - from $87.9 \%$ to $33.7 \%$ and from $75.9 \%$ to $40.9 \%$, respectively. In the test group PPD decreased from $4.58 \mathrm{~mm}$ to $3.15 \mathrm{~mm}$, the CAL gain was $1.37 \mathrm{~mm}$ and the reduction of bleeding and plaque was from $85.6 \%$ to $25.9 \%$ one month after the treatment. The results were statistically significant in favour of the laser group $(p<0.05)$. Microbiological analysis revealed that the pathogens from the red complex decreased 3 times in the control and 6 times in the test group. Therefore, the Er:YAG laser demonstrate pronounced early effectiveness in chronic periodontitis treatment and would be appropriate alternative of the conventional periodontal therapy.

Key words: periodontitis, Er:YAG laser, clinical effectiveness 


\section{INTRODUCTION}

hronic periodontitis is defined as an inflammatory and destructive disease. It is a result of a complex polymicrobial infection and disturbed

homeostasis in susceptible to the disease subjects [1]. Therefore, the aim of its treatment is to remove dental biofilm and calculus and to ensure biocompatible root surfaces [2].

In recent years, a widely discussed alternative treatment of chronic periodontitis, are lasers. There are many types of lasers like $\mathrm{Er}$ : YAG, $\mathrm{CO}_{2}, \mathrm{Nd}$ : YAG, diode, and others, that are used in various dental treatments [3-5]. The Er:YAG laser is the most promising laser in periodontal treatment because of its wavelength of $2940 \mathrm{~nm}$. It is well absorbed by water molecules and hydroxyapatite, as the peak of the absorption of water is slightly less than $3000 \mathrm{~nm}$. Due to this characteristic, the Er:YAG laser is the only one laser system that can ablate not only soft, like the other lasers, but also hard tissues [6-8].

Clinical studies investigating the Er: YAG laser demonstrate its effectiveness in surgical and nonsurgical periodontal treatment, but they do not define benefits compared to the conventional therapy of chronic periodontitis $[9,10]$.

Several in vitro and in vivo studies observe the antimicrobial effectiveness of the Er: YAG laser and report its bactericidal effect against specific periodontal pathogens [10-12]. Nevertheless there is no evidence of beneficial antibacterial effect of the Er: YAG laser compared to conventional therapy of chronic periodontitis [9, 11-14].

Therefore the purpose of the present study is to investigate the early clinical effectiveness of Er:YAG laser in association with the bacteria from the red complex and to compare it with conventional hand instrumentation in chronic periodontitis therapy.

\section{MATERIALS AND METHODS}

30 patients aged from 25 to 62 years $(46.87 \pm 8.496)$ with moderate chronic periodontitis were included in the study (1099 periodontal sites). Inclusion criteria were: probing pocket depths between $4 \mathrm{~mm}$ and $6 \mathrm{~mm}$, loss of clinical attachments up to $4 \mathrm{~mm}$, gingival bleeding upon probing, initial furcation involvement $(\mathrm{F} 1)$ [15], radiographic evidence of bone loss up to $1 / 2$ of the root length, possible tooth mobility [16]. Exclusion criteria were: periodontal treatment in the last 12 months, antibiotic treatment in the last 6 months, systemic diseases or systemic administration of drugs that can influence the course of treatment, and pregnancy. All of the patients signed an informed consent and the study was approved by the Ethical Review Board of Medical University - Plovdiv. 


\section{TREATMENT}

Patients were treated in a split mouth design where two of the quadrants were randomly allocated to scaling and root planning with Gracey curettes (control treatment) and the contralateral two - to Er:YAG laser debridement (test treatment).

Before subgingival instrumentation supragingival tooth cleaning was performed using ultrasonic scaler and tip for supragingival cleaning (Piezon Master 400, EMS Electro Medical Systems SA, Nyon, Switzerland). After that teeth were polished with rubber cups and abrasive polishing paste. Subgingival treatment was accomplished within 24 hours according to the principles of "full-mouth disinfection" [17]. No gingival curettage was performed [18]. All patients were instructed in maintaining a proper oral hygiene regimen.

Control group - hand instrumentation (Gracey curettes)

Patients rinsed with chlorhexidine solution for $30 \mathrm{sec}$ before treatment. Subgingival scaling and root planning was performed with 7 new standart Gracey curettes (Hu-Friedy Mfg. Co., Chicago, IL, USA) using horizontal, vertical and oblique movements until the operator felt smooth and clean root surface.

Test group - laser instrumentation (Er:YAG laser)

Subgingival instrumentation of root surfaces was performed with Er:YAG laser (Lite Touch, Light Instruments Ltd., Yokneam, Israel) using chisel tip with apicocoronal movements in slight angulation against the root surface $\left(15-20^{\circ}\right)$ in contact hard tissue mode. The panel settings were the following: $100 \mathrm{~mJ}, 15 \mathrm{~Hz}, 5-6$ water spray level. After that periodontal pocket debridement was performed using tip 0.6 x $17 \mathrm{~mm}$ and energy settings: $50 \mathrm{~mJ}$ and $30 \mathrm{~Hz}$ in non contact soft tissue mode. Instrumentation ended when the operator felt smooth and clean root surface (Fig. 1).

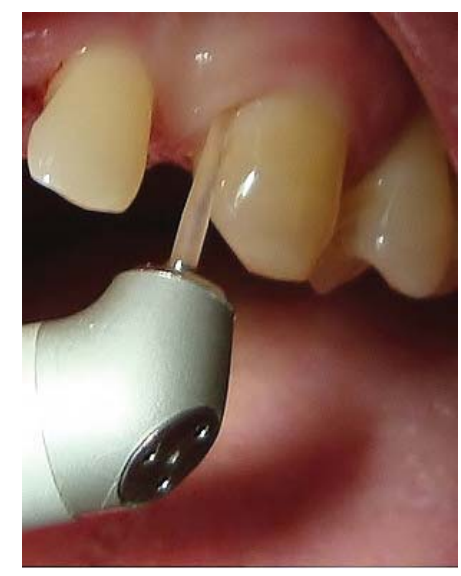

Fig. 1. Root surface debridement with Er:YAG laser

Clinical assessment

Clinical data were measured and recorded using Florida probe (Florida Probe Corporation, Gainesville, FL, USA) at baseline and one month after the therapy (Fig. 
2). Investigated parameters were PPD - probing pocket depth, GR - gingival recession, CAL - clinical attachment level and BOP - bleeding on probing, which were measured in 6 points around each included in the study tooth. Plaque presence/ absence was recorded at 4 points around the tooth $[19,20]$.

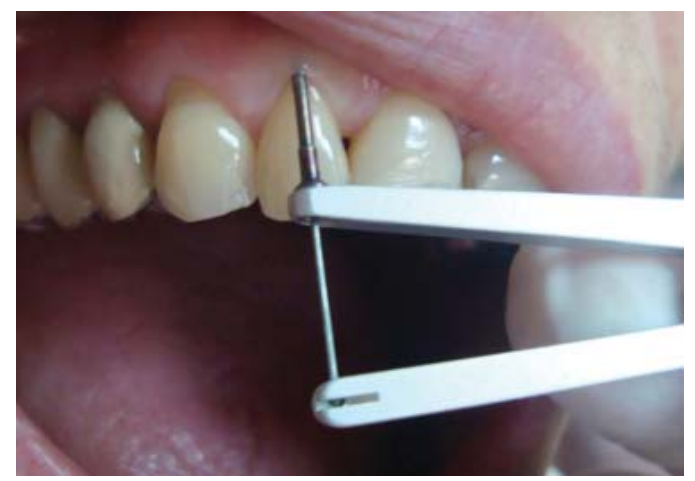

Fig. 2. Clinical examination with Florida probe

\section{Microbiological assessment}

Plaque samples were taken at baseline and one month after therapy from the deepest pocket of each quadrant from 20 randomly selected of all included patients $(n=80)$. Supragingival plaque was carefully removed using curette or sterile cotton. The selected tooth was isolated with sterile paper roll and sterile paper point was inserted in the pocket ( $\varphi=50$, ISO) (Fig. 3). After 20-25 seconds the paper points were placed in a transporting plastic box which was delivered from the laboratory (MIP Pharma $\mathrm{GmbH}$, Germany). Microbiological analysis was performed using real time PCR for determination of specific periodontal pathogens in the sample. In the current study only bacteria from the red complex were presented [21] - (P. Gingivalis - P.g.), Tannerella forsythia (T. Forsythia - T.f.), Prevotela intermedia (P. Intermedia - P.i.).

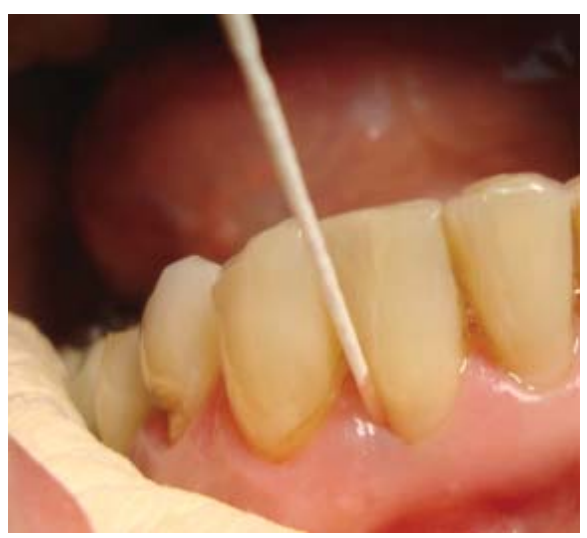

Fig. 3. Microbiological examination 


\section{Statistical analysis}

Data were analyzed using Student $t$ test, Mann-Whitney $U$ test and the results were considered to be significant when $p<0.05$.

\section{RESULTS}

One month after the therapy there was a significant reduction in all clinical parameters in both treatment modalities (Table 1 and Figs. 4 and 5).

Table 1. Changes in PPD, GR and CAL one month after the therapy in both groups

\begin{tabular}{|l|c|c|c|c|c|c|c|c|c|}
\hline & \multicolumn{2}{|c|}{ PPD } & Sig. & \multicolumn{2}{c|}{ GR } & Sig. & \multicolumn{2}{c|}{ CAL } & Sig. \\
\hline & base & 1 month & & base & 1 month & & base & 1 month & \\
\hline Control $(n=527)$ & $4.59 \pm 0.68$ & $3.36 \pm 1.09$ & 0.00 & $0.09 \pm 0.41$ & $0.22 \pm 0.61$ & 0.00 & $4.66 \pm 0.74$ & $3.56 \pm 1.21$ & 0.00 \\
\hline $\begin{array}{l}\text { Test } \\
(n=572)\end{array}$ & $4.58 \pm 0.69$ & $3.15 \pm 1.08$ & 0.00 & $0.14 \pm 0.49$ & $0.18 \pm 0.52$ & 0.02 & $4.71 \pm 0.78$ & $3.34 \pm 1.56$ & 0.00 \\
\hline Sig & 0.83 & 0.00 & & 0.95 & 0.32 & & 0.28 & 0.00 & \\
\hline
\end{tabular}

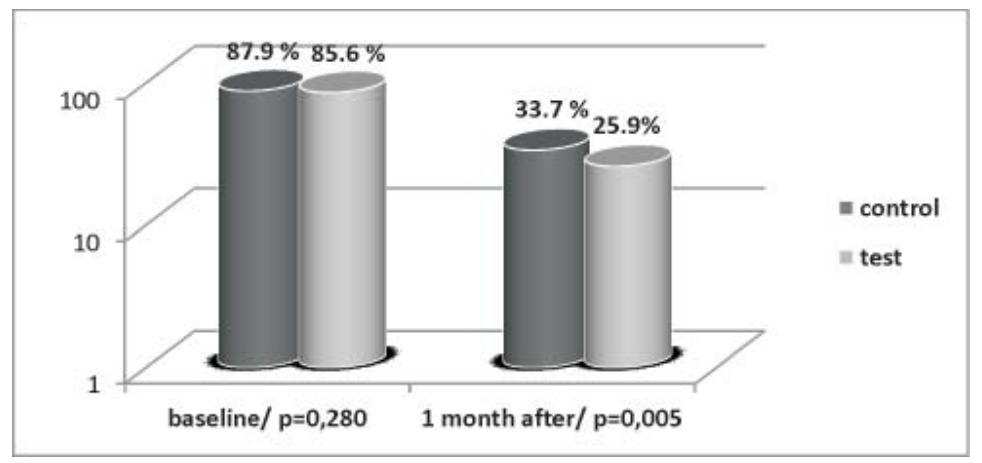

Fig. 4. Changes in bleeding on probing one month after the therapy in both groups

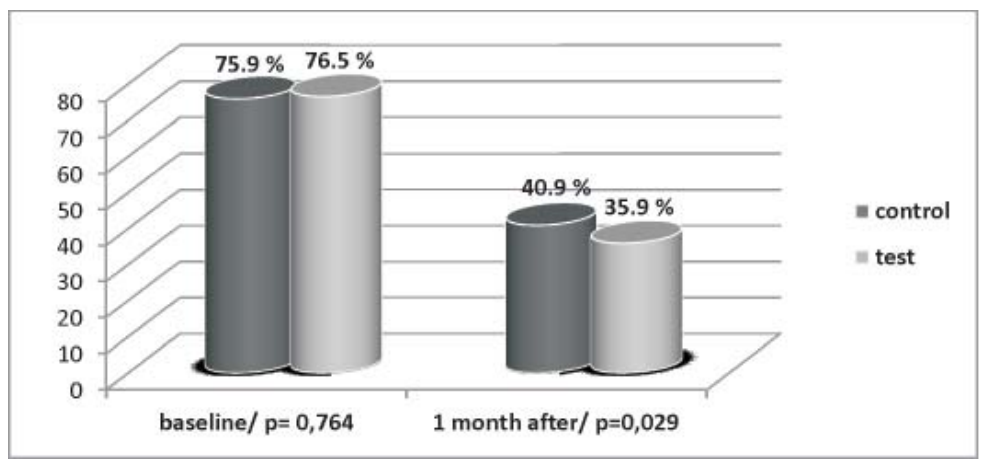

Fig. 5. Changes in plaque presence one month after the therapy in both groups 
The results from the microbial testing for the red complex of bacteria are presented in Table 2. They revealed significant reduction in the red complex one month after the therapy in both groups.

Table 2. Comparison of the red complex at baseline and one month after therapy in both groups

\begin{tabular}{|c|c|c|c|c|c|c|c|c|c|}
\hline & \multirow{2}{*}{$\begin{array}{c}\text { Treatment } \\
\text { group }\end{array}$} & \multirow{2}{*}{ Examination } & \multicolumn{7}{|c|}{ Parameters } \\
\hline & & & $\mathrm{N}$ & $\bar{x}$ & \pm & $\bar{x}_{\mathrm{S}}$ & Sx & Z & $p$ \\
\hline \multirow{2}{*}{$\begin{array}{l}\text { Red } \\
\text { complex }\end{array}$} & \multirow{2}{*}{ Control } & Baseline & 17 & 302887,64 & \pm & 54939,42 & 226521 & \multirow{2}{*}{3.195} & \multirow{2}{*}{0.001} \\
\hline & & 1 month & 17 & 101784,11 & \pm & 29569,53 & 121918 & & \\
\hline \multirow{2}{*}{$\begin{array}{l}\text { Red } \\
\text { complex }\end{array}$} & \multirow{2}{*}{ Test } & Baseline & 15 & 627519,33 & \pm & 299078 & 115832 & \multirow{2}{*}{2.045} & \multirow{2}{*}{0.041} \\
\hline & & 1 month & 15 & 98886,00 & \pm & 32898,38 & 127415 & & \\
\hline
\end{tabular}

\section{DISCUSSION}

The results of the present study demonstrate that the Er:YAG laser has some advantages over conventional non-surgical treatment of moderate chronic periodontal disease with hand instruments. One month after the Er:YAG laser treatment PPD decrease from $4.58 \pm 0.69 \mathrm{~mm}$ to $3.15 \pm 1.08 \mathrm{~mm}$, and the gain of clinical attachments was around $1.37 \mathrm{~mm}$. These results are concordant with the results of some other clinical studies, discussed by Cobb et al [9], where a significant reduction of clinical parameters in the early healing period is seen. Significant reduction in the pocket depth from $4.59 \pm 0.68 \mathrm{~mm}$ to $3.36 \pm 1.09 \mathrm{~mm}$ and gain of clinical attachment around $1.09 \mathrm{~mm}$ was observed also in the hand instrumented group.

There is a significant difference between both groups for all clinical parameters in favor of the Er:YAG laser $(p<0.05)$. Only for the gingival recession there is no difference between the groups. This may be a result from the "phototermal" killing of periodontal bacteria which leads also to reduction of the inflammation. The principle of Er:YAG laser ablation is through evaporation of water molecules and microexplosions within the tissues which leads to its "termomechanical" or "photomechanical" removal. In 2011, Akiyama et al. demonstrate that the bactericidal effect of the Er:YAG laser is also a result from this evaporation [22]. Several clinical studies investigated the antibacterial effect of the laser against specific periodontal pathogens [11-13]. Some of them fail to demonstrate any advantages of the laser over conventional non-surgical hand therapy. The present study shows that the Er: YAG laser periodontal treatment leads to significant reduction in the red complex of periodontal bacteria.

The results of the present clinical and microbiological study proved that the Er:YAG laser possesses a well-defined effectiveness in the treatment of 
moderate chronic periodontitis in the early healing period, which would be a prerequisite for long-term positive therapeutic outcomes. In order to provide more significant results after Er:YAG laser treatment, further investigation is needed in the future.

\section{REFERENCES}

1. S anz, M. et al. Periodontal infections: understanding the complexity--consensus of the Seventh European Workshop on Periodontology. - J. Clin. Periodontol., 38, 2011, Suppl. 11, 3-6.

2. Comprehensive periodontal therapy: a statement by the American Academy of Periodontology. - J. Periodontol., 82, 2011, № 7, 943-949.

3. Romanos, G. E. The state of the science of lasers in dentistry. - J. Dent. Hyg., 86, 2012, № 1, 9-10.

4. Coluzzi, D. J. et R. A. Convissar. Lasers in clinical dentistry. - Dent. Clin. North Am., 48, 2004, № 4, 11-12.

5. La la b o nova, H., G. Todorov et H. Daskalov. Clinical assessment of the effect of low level laser treatment of oral mucosa decubitus ulcers. - J. of IMAB, 19, 2013, № 4, 380-382.

6. A o ki , A. et al. Lasers in nonsurgical periodontal therapy. - Periodontol., 36, 2004, 59-97.

7. S chwarz, F. et al. Laser application in non-surgical periodontal therapy: a systematic review. - J. Clin. Periodontol., 35, 2008, (8 Suppl.), 29-44.

8. I s hi kaw a , I., A. Aoki et A. A. Takasaki. Potential applications of Erbium:YAG laser in periodontics. - J. Periodont. Res., 39, 2004, 275-285.

9. C o b b , C. M., S. B. Low et D. J. Coluzzi. Lasers and the treatment of chronic periodontitis. - Dent. Clin. North Am., 54, 2010, № 1, 35-53.

10. S gola stra, F. et al. Efficacy of Er:YAG laser in the treatment of chronic periodontitis: systematic review and meta-analysis. - Lasers Med. Sci., 27, 2012, № 3, 661-673.

11. Tomasi, C. et al. Short-term clinical and microbiologic effects of pocket debridement with an Er:YAG laser during periodontal maintenance. - J. Periodontol., 77, 2006, № 1, 111-118.

12. Derdilopoulou, F. V. et al. Microbiological findings after periodontal therapy using curettes, Er:YAG laser, sonic, and ultrasonic scalers. - J. Clin. Periodontol., 34, 2007, № 7, 588-598.

13. Lopes, B. M. et al. Clinical and microbiologic follow-up evaluations after non-surgical periodontal treatment with erbium:YAG laser and scaling and root planing. - J. Periodontol., 81, 2010, № 5, 682-691.

14. Ko m a t s u , Y., T. Morozumi et D. Abe. Effects of Erbium-Doped: Yttrium Aluminum Garnet (Er:YAG) laser on bacteremia due to scaling and root planning. - J. Lasers Med. Sciences, 3, 2012, № 4, 175-184.

15. Hamp, S. E., S. Nyman et J. Lindhe. Periodontal treatment of multirooted teeth. Results after 5 years. - J. Clin. Periodontol., 2, 1975, 126.

16. P a r a m e ter on chronic periodontitis with slight to moderate loss of periodontal support. American Academy of Periodontology. - J. Periodontol., 71, 2000, Suppl. 5, 853-855.

17. Quiry ne n, M. et al. Benefit of "one-stage full-mouth disinfection" is explained by disinfection and root planing within 24 hours: a randomized controlled trial. - J. Clin. Periodontol., 33, 2006, № 9, 639-647. 
18. The American Academy of Periodontology statement regarding gingival curettage. - J. Periodontol., 73, 2002, № 10, 1229-1230.

19. O ' L e a ry, T. J., R. B. Drake et J. E. Naylor. The plaque control record. - J. Periodontol., 43, 1972, № 1,38 .

20. A in a m o, J. et I. Bay I. Problems and proposals for recording gingivitis and plaque. Internati

21. So cransky, S. S. et al. Microbial complexes in subgingival plaque. - J. Clin. Periodontol., 25, 1998, № 2, 134-144.

22. A ki y a m a, F. et al. In vitro studies of the ablation mechanism of periodontopathic bacteria and decontamination effect on periodontally diseased root surfaces by erbium:yttrium-aluminum-garnet laser. - Lasers Med. Sci., 26, 2011, № 2, 193-204.

- Corresponding author:

Blagovesta Yaneva, PhDc

Department of Periodontology and Oral Diseases

Faculty of Dental Medicine

Medical University - Plovdiv

3 Hristo Botev Blvd.

$\mathrm{Bg}-4000$ Plovdiv

(J) +359888539588

e-mail: b_yaneva@yahoo.com 